\title{
Dust-ion-acoustic Solitary Waves and Their Instability in a Magnetized Adiabatic Dusty Electronegative Plasma
}

\author{
O. Rahman and M. M. Haider \\ Department of Physics, Mawlana Bhashani Science and Technology University, Santosh, Tangail, Bangladesh \\ Email: armanphy203@gmail.com
}

\begin{abstract}
The basic features of a nonlinear obliquely propagating dust-ion-acoustic (DIA) solitary waves (SWs) in a magnetized adiabatic dusty electronegative plasma (MADENP) containing Maxwellian electrons, vortex-like (trapped) negative ions, positively charged stationary dust, and adiabatic mobile inertial positive ions have been theoretically investigated. The well known modified Zakharov Kuznetsov (mZK) is derived by using the reductive perturbation technique. The dependence of solitary wave structures on positive ion adiabaticity, external magnetic field (obliqueness), trapped negative ions, and positively charged stationary dust are studied explicitly. The three-dimensional instability of this DIASWs is also analyzed using small- $k$ perturbation expansion technique. It is found that the nature of DIASW, the instability criterion and the growth rate of unstable perturbations are significantly modified by the external magnetic field, the propagation directions of both the nonlinear waves and their perturbation modes.
\end{abstract}

Keywords: Dust-ion-acoustic solitary waves, adiabatic dusty plasma, trapped negative ions.

\section{Introduction}

It is well known that the presence of statically charged dust grains in a plasma can modify the behavior of the usual existing plasma wave spectra and instabilities [1,2,3,4,5]. Bliokh and Yaroshenko [1] have studied electrostatic waves in dusty plasmas and applied their results in interpreting spoke-like structures in Saturn's rings. Angelis et al. [2] have studied the propagation of ion acoustic waves in the presence of massive charged dust particles in a dusty plasma and they have derived an equation describing low-frequency electrostatic perturbations on a non-homogeneous background, where the inhomogeneity is due to a distribution of charge grains, each surrounded by an equilibrium statistical distribution of plasma particles and applied their results in interpreting the low-frequency noise enhancement in the dusty regions of Halley's Comet. Shukla and Silin [5] have first theoretically shown that due to the conservation of equilibrium charge density a dusty plasma supports low-frequency DIA waves whose phase speed is much smaller (larger) than electron (ion) thermal speed. Barkan et al. [6] have also experimentally observed the DIA waves. The dust grains are charged due to a variety of processes $[4,7,8]$. The dust grains of different sizes can acquire different polarities; large grains become negatively charged and small ones become small positively charged $[9,10,11]$. These positively charged dust particles have been found in different regions of space, viz. cometary tails [9,10,11], Jupiter's magnetosphere [12], etc. There are three principal mechanisms by which a dust grain becomes positively charged [13]. These are photoemission in the presence of a flux of ultraviolet (UV) photons, thermionic emission induced by radiative heating, and secondary emission of electrons from the surface of the dust grains.

Sayed et al. [14] have studied the DIASWs in an unmagnetized multi-ion dusty plasma containing inertia-less isothermal electrons, cold inertial positive and negative ions, and negatively charged static dust. Rahman et al. [15] have theoretically studied the basic properties (phase speed, amplitude, and width) and multidimensional instability of the obliquely propagating DIASWs in a magnetized four components dusty electronegative plasmas which contains isothermal electrons, non-isothermal trapped negative ions, cold mobile positive ions, and arbitrarily charged stationary dust by deriving mZK equations instead of usual ZK equations due to trapped negative ions. Kundu et al. [16] have investigated the nonlinear propagation of the obliquely propagating DIASWs and multidimensional instability in a four-component magnetized dusty electronegative plasmas and found that the effects of nonthermal negative ions significantly modify the nature of the DIASWs structures in a dusty plasma. Haider et al. [17] have also studied the DIA 
SWs waves in multi-component magnetized dusty plasmas which consist of negative and positive ions, Boltzmann distributed hot electrons, and immobile negative and positive dust grains. Haider et al. [18] have also theoretically investigated the nonlinear propagation of DIA solitary structures in a magnetized dusty plasma consisting negatively and positively charged ion fluid, trapped electrons following a vortexlike distribution, and arbitrary charged stationary dust where restoring force provided by the thermal pressure of electrons and the inertia is due to the ion mass for instability analysis. Mamun [19] has theoretically investigated the basic properties of one-dimensional DIASWs in an unmagnetized adiabatic dusty plasma system which contains inertialess adiabatic electrons, inertial adiabatic ions, and negatively charged stationary dust particles. Duha et al. [20] have considered adiabatic effect in Maxwellians negative ions and they have found both positive and negative SWs potential due to the effect of the Maxwellian negative ion. But to the best of our knowledge till now the effect of the adiabaticity of ions in magnetized plasmas has not been studied, which can significantly modify the basic features of DIASWs. We hope the findings of the study may be helpful in understanding some astrophysical $[21,22,23]$ and laboratory $[24,25,26]$ plasmas. Therefore in our present work, we have considered a MADENP containing Boltzmann electrons, trapped negative ions, adiabatic mobile positive ions, and positively charged stationary dust in the presence of an external static magnetic field, and study the properties of the obliquely propagating DIASWs and their multidimensional instability criterion.

The manuscript is organized as follows. The governing equations are presented in section 2 . The mZK equation is derived by employing the reductive perturbation method in section 3. The SW solution of this mZK equation is obtained and the properties of these DIA solitary structures are discussed in section 4 . The instability criterion is analyzed in section 5. A brief discussion is, finally, presented in section 6 .

\section{Governing Equations}

The nonlinear dynamics of the low-frequency electrostatic perturbation mode in such a MADENP system is described by

$$
\begin{aligned}
& \frac{\partial n_{i}}{\partial t}+\nabla \cdot\left(n_{i} \mathbf{u}_{i}\right)=0 \\
& \frac{\partial \mathbf{u}_{i}}{\partial t}+\left(\mathbf{u}_{i} \cdot \nabla\right) \mathbf{u}_{i}=-\nabla \phi+\omega_{c p} \mathbf{u}_{i} \times \hat{k}-\frac{\sigma_{i}}{n_{i}} \nabla p_{i} \\
& \frac{\partial p_{i}}{\partial t}+\left(\mathbf{u}_{i} \cdot \nabla\right) p_{i}+\gamma p_{i} \nabla \cdot \mathbf{u}_{i}=0 \\
& \nabla^{2} \phi=\mu_{e} e^{\phi}+\mu_{n} n_{n}-n_{i}-\mu_{d}
\end{aligned}
$$

where $n_{i}\left(n_{n}\right)$ is the positive (negative) ion number density normalized by $n_{i 0}\left(n_{n o}\right), u_{i}$ is the positive ion fluid speed normalized by the positive ion-acoustic speed $C_{i}=\left(k_{B} T_{e} / m_{i}\right)^{1 / 2}, \phi$ is the electrostatic wave potential normalized by $k_{B} T_{e} / e, \mu_{n}=\left(1-\mu_{e}+\mu_{d}\right), \mu_{d}=Z_{d} n_{d 0} / n_{i 0}, \mu_{e}=n_{e 0} / n_{i 0}$, and $m_{i}$ is the mass of positive ion. $\gamma=C_{p}^{i} / C_{v}^{i}$, where $C_{p}^{i}\left(C_{v}^{i}\right)$ is the specific heat of the ion at constant pressure (volume), $\omega_{c i}=\left(e B_{0} / m_{i}\right) / \omega_{p i}$ is the positive ion cyclotron frequency normalized by the positive ion plasma frequency $\omega_{p i}=\left(4 \pi e^{2} n_{i 0} / m_{i}\right)^{1 / 2}, p_{i}$ is the positive ion pressure normalized by $n_{i 0} k_{B} T_{i 0}$. The time variable $t$ is normalized by the positive ion plasma period $\omega_{p i}^{-1}$ and the space variable is normalized by $\lambda_{D}$. The inertialess negative ion has a non-Maxwellians characteristics described by a vortex-like velocity distribution which leads to a negative number density of the form $[27,28,29,30,31,32]$,

$$
n_{n}=1+\sigma_{n} \phi-\frac{4}{3} Q \sigma_{n}^{\frac{3}{2}} \phi^{\frac{3}{2}}+\frac{1}{2} \sigma_{n}^{2} \phi^{2}
$$

where $Q=(1-\beta) / \sqrt{\pi}, \sigma_{n}=T_{e} / T_{n}, T_{e}\left(T_{n}\right)$ is the electron (negative ion) temperature. $\beta$ is a parameter which determines the number of trapped negative ions [33,34] and it is defined as the ratio of the free negative ion temperature $T_{i f}$ to the trapped negative ion temperature $T_{i t}[33,34]$. We note that $\beta=1$ $(\beta=0)$ represents a Maxwellian (flat-topped) negative ion distribution, whereas $\beta<0$ represents a vortex-like excavated trapped negative ion distribution. We are interested in $\beta<0$. 


\section{Derivation of mZK Equation}

To derive the well-known mZK equation by using the reductive perturbation technique, we consider the following the stretched coordinates $[27,29,35]$.

$$
\left.\begin{array}{l}
X=\epsilon^{1 / 4} x \\
Y=\epsilon^{1 / 4} y \\
Z=\epsilon^{1 / 4}\left(z-v_{p} t\right) \\
\tau=\epsilon^{3 / 4} t
\end{array}\right\}
$$

where $\epsilon$ is a small parameter measuring the weakness of the dispersion, $v_{p}$ is the unknown phase speed normalized by the positive ion-acoustic speed $\left(C_{p}\right)$. We further expand the perturbed quantities $n_{i}, u_{i}, p_{i}$ and $\phi$ about their equilibrium values as [36]

$$
M=M^{(0)}+\sum_{n=1}^{\infty} \epsilon^{(n+1) / 2} M^{(n)}
$$

where $M=\left[n_{i}, p_{i}, u_{i z}, \phi\right]$ and $M^{(0)}=[1,1,0,0]$, On the other hand

$$
u_{i x, y}=\sum_{n=1}^{\infty} \epsilon^{(n+1) / 4)} u_{i x, y}^{(n)}
$$

To obtain the set of lowest order equations from Eqns. (1)-(3), we substituted Eqns. (5)-(8) into Eqns. (1)-(3) and obtained.

$$
\begin{aligned}
n_{i}^{(1)} & =\frac{\phi^{(1)}}{v_{p}^{2}-\gamma \sigma_{i}}, \quad p_{i}^{(1)}=\frac{\gamma}{v_{p}^{2}-\gamma \sigma_{i}} \phi^{(1)} \\
u_{i z}^{(1)} & =\frac{v_{p}}{v_{p}^{2}-\gamma \sigma_{i}} \phi^{(1)} \\
u_{i x}^{(1)} & =-\frac{R}{\omega_{c i}} \frac{\partial \phi^{(1)}}{\partial Y}, \quad u_{i y}^{(1)}=\frac{R}{\omega_{c i}} \frac{\partial \phi^{(1)}}{\partial X}
\end{aligned}
$$

where $R=v_{p}^{2} /\left(v_{p}^{2}-\gamma \sigma_{i}\right)$. The lowest order Poisson's equation can be found as,

$$
\mu_{e} \phi^{(1)}+\mu_{n} \sigma_{n} \phi^{(1)}=n_{i}^{(1)}
$$

The following linear dispersion relation for this MADENP system can be obtained using the value of $n_{i}^{(1)}$ from eqn. (9) into eqn. (12).

$$
v_{p}=\sqrt{\frac{1}{\mu_{e}+\mu_{n} \sigma_{n}}+\gamma \sigma_{i}}
$$

Similarly, to the next higher order of $\epsilon$, we obtain the second-order $\mathrm{x}$ - and $\mathrm{y}$-components of the momentum equation, and Poisson's equation as

$$
\begin{aligned}
& u_{i x}^{(2)}=\frac{v_{p} R}{\omega_{c i}^{2}} \frac{\partial^{2} \phi^{(1)}}{\partial X \partial Z}, \quad u_{i y}^{(2)}=\frac{v_{p} R}{\omega_{c i}^{2}} \frac{\partial^{2} \phi^{(1)}}{\partial Y \partial Z} \\
& {\left[\frac{\partial^{2}}{\partial X^{2}}+\frac{\partial^{2}}{\partial Y^{2}}+\frac{\partial^{2}}{\partial Z^{2}}\right] \phi^{(1)}=\left(\mu_{e}+\mu_{n} \sigma_{n}\right) \phi^{(2)}-\frac{4}{3} Q \mu_{n} \sigma_{n}^{3 / 2}\left[\phi^{(1)}\right]^{3 / 2}-n_{i}^{(2)}}
\end{aligned}
$$

Again, following the same procedure, one can obtain the next higher order continuity equation, pressure equation, and z-component of momentum equation as

$$
\frac{\partial n_{i}^{(1)}}{\partial \tau}-v_{p} \frac{\partial n_{i}^{(2)}}{\partial Z}+\frac{\partial u_{i x}^{(2)}}{\partial X}+\frac{\partial u_{i y}^{(2)}}{\partial Y}+\frac{\partial u_{i z}^{(2)}}{\partial Z}=0
$$




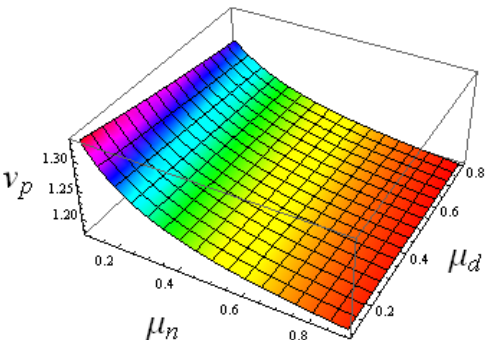

(a)

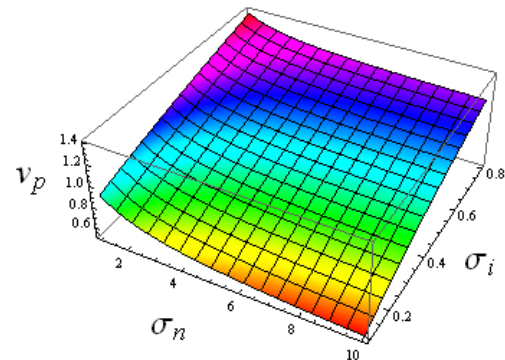

(b)

Figure 1. (Color online) Variation of the phase speed $\left(v_{p}\right)$ of the SWs: (a) with $\mu_{n}$ and $\mu_{d}$ for $\gamma=1.6, \sigma_{n}=10$, $\sigma_{i}=0.8$, and (b) with $\sigma_{n}$ and $\sigma_{i}$ for $\gamma=1.6, \mu_{n}=0.9$ and $\mu_{d}=0.5$.

$$
\begin{aligned}
& \frac{\partial u_{i z}^{(1)}}{\partial \tau}-v_{p} \frac{\partial u_{i z}^{(2)}}{\partial Z}+\frac{\partial \phi^{(2)}}{\partial Z}+\sigma_{i} \frac{\partial p_{i}^{(2)}}{\partial Z}=0 \\
& \frac{\partial p_{i}^{(1)}}{\partial \tau}-v_{p} \frac{\partial p_{i}^{(2)}}{\partial Z}+\gamma \frac{\partial u_{i x}^{(2)}}{\partial X}+\gamma \frac{\partial u_{i y}^{(2)}}{\partial Y}+\gamma \frac{\partial u_{i z}^{(2)}}{\partial Z}=0
\end{aligned}
$$

Now, using eqns. (9)-(18), we can obtain

$$
\frac{\partial \phi^{(1)}}{\partial \tau}+A B \sqrt{\phi^{(1)}} \frac{\partial \phi^{(1)}}{\partial Z}+\frac{1}{2} A \frac{\partial}{\partial Z}\left[\frac{\partial^{2}}{\partial Z^{2}}+D\left(\frac{\partial^{2}}{\partial X^{2}}+\frac{\partial^{2}}{\partial Y^{2}}\right)\right] \phi^{(1)}=0
$$

where

$$
\begin{aligned}
& A=\frac{v_{p}^{3}}{R^{2}} \\
& B=Q \mu_{n} \sigma_{n}^{3 / 2} \\
& D=1+\frac{R^{2}}{\omega_{c i}^{2}}
\end{aligned}
$$

Equation (19) is a modified mZK equation in three dimensions, exhibiting a stronger nonlinearity, smaller width, and larger propagation velocity of the nonlinear wave.

\section{SW Solution of the mZK Equation}

To study the properties of the SWs propagating in the present system we follow the same procedure as [15] to get the steady state solution of the well-known mZK equation. Therefore, after some iterative mathematical calculation (not shown here), one can obtain:

$$
\phi_{0}(Z)=\phi_{m} \operatorname{sech}^{4}(\kappa Z)
$$

where the amplitude and width of the SWs are represented respectively as

$$
\begin{aligned}
& \phi_{m}=\left[15 u_{0} / 8 \delta_{1}\right]^{2} \\
& \Delta=\sqrt{16 \delta_{2} / u_{0}}
\end{aligned}
$$

with

$$
\begin{aligned}
\delta_{1} & =A B \cos \delta \\
\delta_{2} & =\frac{1}{2} A\left(\cos ^{3} \delta+D \sin ^{2} \delta \cos \delta\right)
\end{aligned}
$$




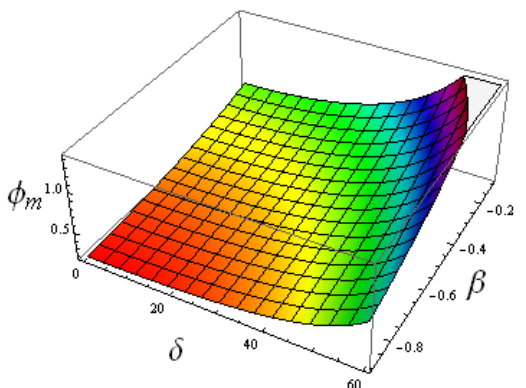

(a)

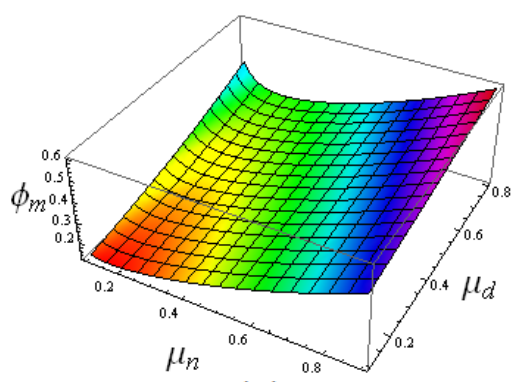

(c)

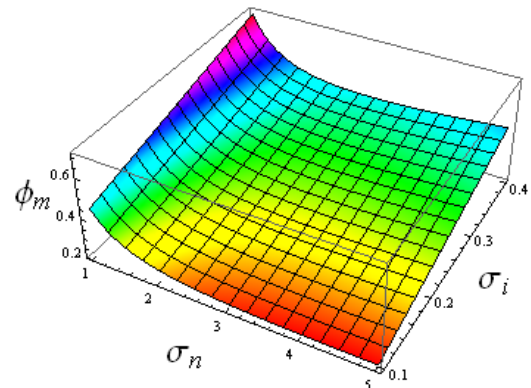

(b)

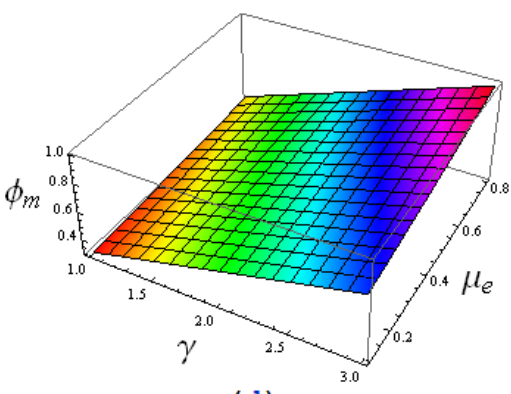

(d)

Figure 2. (Color online) Variation of the amplitude $\left(\phi_{m}\right)$ of the SWs: (a) with $\delta$ and $\beta$ for $u_{0}=0.1, \gamma=1.6$, $\mu_{n}=0.9, \mu_{e}=0.5, \sigma_{i}=0.3$ and $\sigma_{n}=10$, (b) with $\sigma_{n}$ and $\sigma_{i}$ for $\beta=-0.3$, and $\delta=30^{\circ}$, (c) with $\mu_{n}$ and $\mu_{d}$ for $\beta=-0.3$ and $\delta=30^{\circ}$, and (d) with $\gamma$ and $\mu_{e}$ for $\beta=-0.3$ and $\delta=30^{\circ}$. The other parameters are the same as those used in Fig. 2(a).

It has been found that the amplitude of the SWs is proportional to the square of the soliton speed $u_{0}$ and the width is inversely proportional to square root of this soliton speed. That is the profile of the faster soliton will be spikier than a slower one.

It has also been clear from the (24) that the SWs will always associate with positive potential $\left(\phi_{m}>0\right)$, due to the effect of trapped negative ions. This result completely agrees with Rahman et al. [15] but they have not considered adiabatic effect. The different results have been found by Duha et al. [20] where negative and positive solitary waves are found. Figs. 1(a) and (b) show how the phase speed of SWs varies with various parameters. Fig. 1(a) indicates that the phase speed of SWs decreases with increasing the values of both $\mu_{n}$ and $\mu_{d}$. It means that the SWs propagate slowly with increasing positive dust as well as negative ion number density. Fig. 1(b) shows the variation of $v_{p}$ with $\sigma_{n}$ and $\sigma_{i}$. From this figure, we have seen that the phase speed of SWs decreases with $\sigma_{n}$ but increases with $\sigma_{i}$. Figs. 2(a)-(d) show the variation of SWs with $\delta, \beta, \sigma_{n}, \sigma_{i}, \mu_{n}, \mu_{d}, \gamma$ and $\mu_{e}$ respectively. Fig. 2(a) indicates that the amplitude of the SW potential increases with increasing the values of both $\delta$ and $\beta$. This result completely agrees with Haider et al. [18]. Fig. 2(b) indicates the amplitude of the SW potential increases with increasing the values of $\sigma_{i}$ but it decreases with $\sigma_{n}$. Fig. 2(c) indicates that the amplitude of the SW potential increases with increasing the values of $\mu_{n}\left(\mu_{d}\right)$ rapidly (slightly). Fig. 2(d) indicates that the amplitude of the SW potential increases with increasing the values of $\gamma\left(\mu_{e}\right)$ rapidly (slightly). It means that effect of adiabatic positive ion changes the potential of SWs, which is found by Haider et al. [18]. The variation of the width $(\Delta)$ for positive SWs with $\mu_{n}, \mu_{d}, \gamma, \omega_{c i}, \sigma_{n}, \sigma_{i}$ and $\delta$ is represented in Figs. 3(a)-(d). It is clear from Fig. 3(a) that the width of the SWs decreases with both of $\mu_{n}$ and $\mu_{d}$. Fig. 3(b) indicates that the width of the SWs increases with increasing the values of $\gamma$ and it decreases with the value of $\omega_{c i}$. Fig. $3(\mathrm{c})$ indicates that the width of the SWs decreases with $\sigma_{n}$ but it increases with $\sigma_{i}$. This result completely agrees with the result that was obtained by Duha et al. [20]. Fig. 3(d) indicates that width of the SWs increases linearly with $\delta$ for the lower range, i.e., from $0^{0}$ to about $60^{\circ}$ but decreases for its higher range, i.e., from about $60^{0}$ to $90^{\circ}$ and as $\delta \rightarrow 90^{\circ}$, the width of the SWs goes to zero. It has been also found that the width of the SWs is larger for $\omega_{c i}=0.1$ red curve and smaller for $\omega_{c i}=0.7$ green curve. It means that the increasing the magnetic field decreases the width of SWs which is valid for $\delta<90^{\circ}$. 


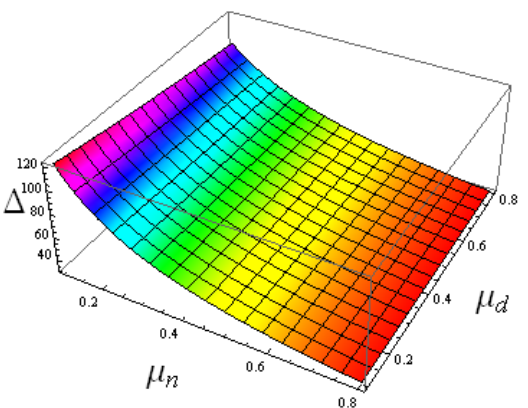

(a)

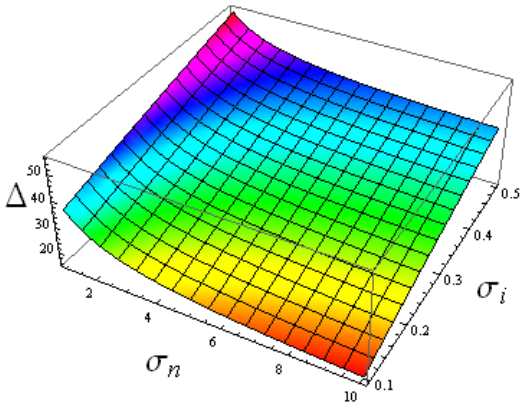

(c)

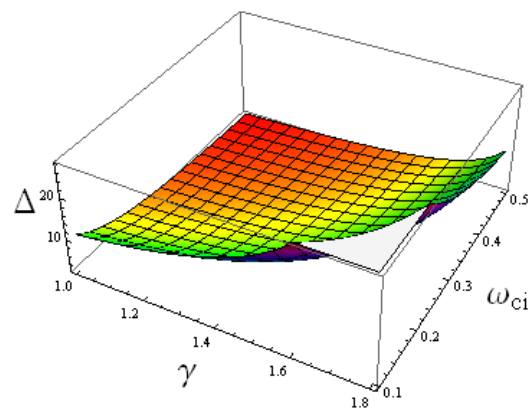

(b)

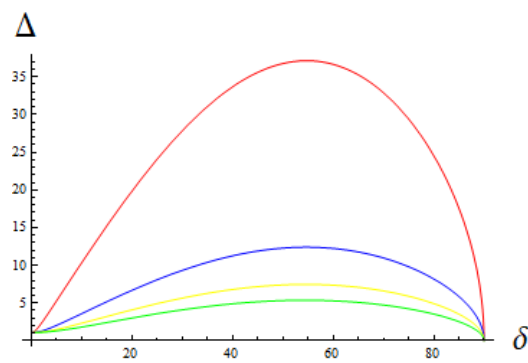

(d)

Figure 3. (Color online) Variation of the width $(\Delta)$ of the SWs: (a) with $\mu_{n}$ and $\mu_{d}$ for $\omega_{c i}=0.1$ and $\delta=30^{\circ}$, (b) with $\gamma$ and $\omega_{c i}$ for $\delta=30^{\circ}$, (c) with $\sigma_{n}$ and $\sigma_{i}$ for $\omega_{c i}=0.1$ and $\delta=30^{\circ}$, and (d) with $\delta$ for $\omega_{c i}=0.1$ (red curve), 0.3 (blue curve), 0.5 (yellow curve) and 0.7 (green curve). The other parameters are the same as those used in Fig. 2(a).

\section{$5 \quad$ Instability Analysis}

In this section, we have investigated the instability condition for the obliquely propagating SWs, discussed in the previous section, by using the small- $k$ expansion technique[37,38,39,40,41]. To do that we first assume that

$$
\phi^{(1)}=\phi_{0}(Z)+\phi(Z, \zeta, \eta, t)
$$

where $Z, \zeta, \eta$ and $t$ are the corresponding components for the new reference frame. In case of a longwavelength plane wave perturbation in a direction with direction cosines, $\left(l_{\zeta}, l_{\eta}, l_{\xi}\right)$, $\phi$ is given by

$$
\phi=\varphi(Z) e^{i\left[k\left(l_{\zeta} \zeta+l_{\eta} \eta+l_{\xi} Z\right)-\omega t\right]}
$$

where, $l_{\zeta}^{2}+l_{\eta}^{2}+l_{\xi}^{2}=1, k$ is the wave vector, and $\omega$ is the wave angular frequency. For small- $k$ technique one can express $\varphi(Z)$ and $\omega$ as:

$$
\begin{aligned}
& \varphi(Z)=\varphi_{0}(Z)+k \varphi_{1}(Z)+k^{2} \varphi_{2}(Z)+\cdots \\
& \omega=k \omega_{1}+k^{2} \omega_{2}+\cdots
\end{aligned}
$$

Now, using the above equations (23)-(29) into equation (19) and following the same procedure as [15], we have obtained the following dispersion relation (after long mathematical calculation not shown here):

$$
\omega_{1}=\Omega-l_{\xi} u_{0}+\left(\Omega^{2}-\Upsilon\right)^{1 / 2}
$$

where

$$
\Omega=\frac{16}{21}\left(\sqrt{\phi_{m}} \mu_{1}-3 \mu_{2} \kappa^{2}\right)
$$




$$
\Upsilon=\frac{512}{945}\left(\phi_{m}^{2} \mu_{1}^{2}-5 \sqrt{\phi_{m}} \mu_{1} \mu_{2} \kappa^{2}-\frac{15}{2} \mu_{2}^{2} \kappa^{4}+30 \delta_{2} \mu_{3} \kappa^{4}\right)
$$

with

$$
\begin{aligned}
& \mu_{1}=\delta_{1} l_{\xi}+\delta_{3} l_{\zeta}, \quad \mu_{2}=3 \delta_{2} l_{\xi}+\delta_{5} l_{\zeta} \\
& \mu_{3}=3 \delta_{2} l_{\xi}^{2}+2 \delta_{5} l_{\zeta} l_{\xi}+\delta_{6} l_{\zeta}^{2}+\delta_{7} l_{\eta}^{2} \\
& \delta_{3}=-A B \sin \delta, \\
& \delta_{5}=A\left[D\left(\sin \delta \cos ^{2} \delta-\frac{1}{2} \sin ^{3} \delta\right)-\frac{3}{2} \sin \delta \cos ^{2} \delta\right] \\
& \delta_{6}=-A\left[D\left(\sin ^{2} \delta \cos \delta-\frac{1}{2} \cos ^{3} \delta\right)-\frac{3}{2} \sin ^{2} \delta \cos \delta\right] \\
& \delta_{7}=\frac{1}{2} A D \cos \delta, \quad \delta_{8}=-\frac{1}{2} A D \sin \delta
\end{aligned}
$$

From the dispersion relation (28), one can easily say that the SWs always become unstable if $\left(\Upsilon-\Omega^{2}\right)>0$. So, we can express the instability condition as

$$
S_{i}>0
$$

with

$$
S_{i}=l_{\eta}^{2}\left[\omega_{c i}^{2}+R^{2} \sin ^{2} \delta\right]+l_{\zeta}^{2}\left[\omega_{c i}^{2}-\frac{9}{7}\left(R^{2}+\omega_{c i}^{2}\right) \tan ^{2} \delta\right]
$$

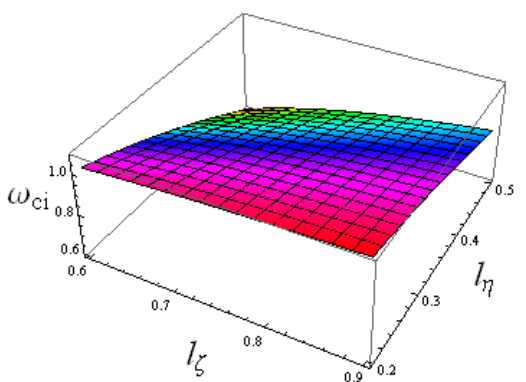

(a)

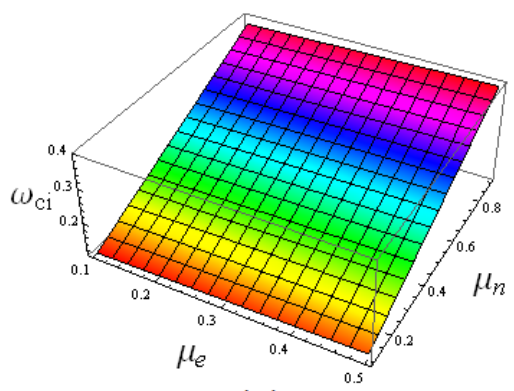

(c)

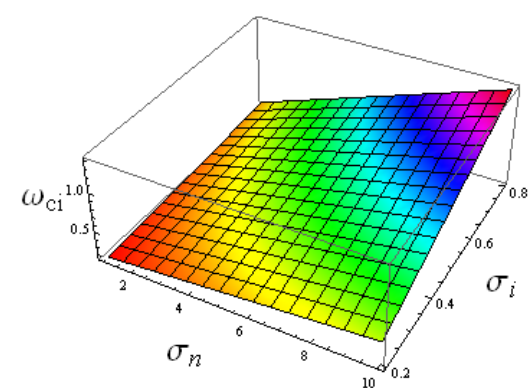

(b)

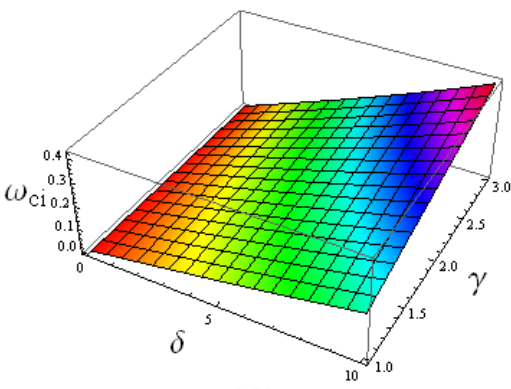

(d)

Figure 4. (Color online) Surface plot $\left(S_{i}=0\right.$ ), variation of $\omega_{c i}$, (a) with $l_{\zeta}$ and $l_{\eta}$ for $\delta=10^{\circ}$, (b) with $\sigma_{n}$ and $\sigma_{i}$ for $\delta=10^{\circ}, l_{\eta}=0.5$, and $l_{\zeta}=0.6$, , (c) with $\mu_{e}$ and $\mu_{n}$ for $\delta=10^{\circ}, l_{\eta}=0.5$, and $l_{\zeta}=0.5$, and (d) with $\delta$ and $\gamma$ for $l_{\zeta}=0.5$ and $l_{\eta}=0.5$ above which the SWs become unstable and below which the SWs become stable. The other parameters are the same as those used in Fig. 2(a).

We have graphically obtained the parametric regimes (values of $\delta, \omega_{c i}, l_{\zeta}, l_{\eta}, \sigma_{i}, \sigma_{n}, \gamma, \mu_{e}$ and $\mu_{n}$ ) for which the SWs become stable and unstable. These are shown in Figs. 4(a)-(d), which indicate that for the parameters above the surface the SWs become unstable and below the surface the SWs become 


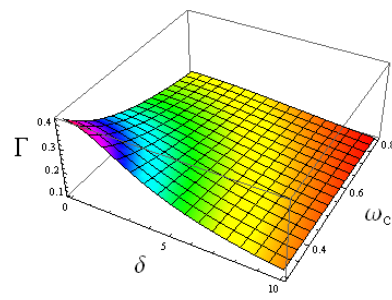

(a)

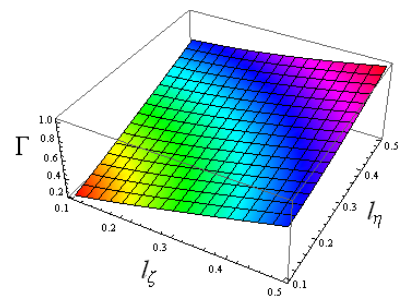

(b)

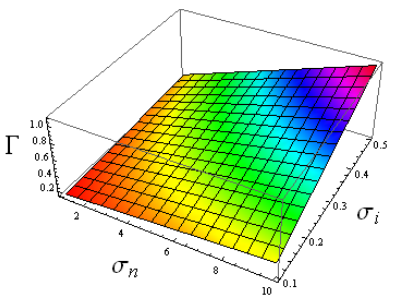

(c)

Figure 5. (Color online) Variation of $\Gamma$ : (a) with $\delta$ and $\omega_{c i}$ for $l_{\zeta}=0.01$, and $l_{\eta}=0.9$, (b) with $l_{\zeta}$ and $l_{\eta}$ for $\omega_{c i}=0.1$ and $\delta=180^{\circ}$, and (c) with $\sigma_{n}$ and $\sigma_{i}$ for $\omega_{c i}=0.1, \delta=180^{\circ}, l_{\zeta}=0.01$, and $l_{\eta}=0.5$. The other parameters are the same as those used in Fig. 2(a).

stable. Fig. 4(a) represents the $S_{i}=0$ surface plot showing variation of $\omega_{c i}$ with $l_{\zeta}$ and $l_{\eta}$. From this it has been found that, as the value of $l_{\zeta}\left(l_{\eta}\right)$ increases, the value of $\omega_{c i}$ for which the SWs become unstable increases (decreases). Fig. 4(b) represents the $S_{i}=0$ surface plot showing variation of $\omega_{c i}$ with $\sigma_{n}$ and $\sigma_{i}$. From this it has been found that, as the value of $\sigma_{n}$ and $\sigma_{i}$ increases, the value of $\omega_{c i}$ for which the SWs become unstable increases. Fig. 4(c) represents the $S_{i}=0$ surface plot showing variation of $\omega_{c i}$ with $\mu_{e}$ and $\mu_{n}$. This indicate that, as the value of $\mu_{e}$ and $\mu_{n}$ increases, the value of $\omega_{c i}$ for which the SWs become unstable also increases. Fig. $4(\mathrm{~d})$ represents the $S_{i}=0$ surface plot showing variation of $\omega_{c i}$ with $\delta$ and $\gamma$. From this it has been found that, as the value of $\delta$ and $\gamma$ increases, the value of $\omega_{c i}$ for which the SWs become unstable increases.

If this instability criterion $S_{i}>0$ is satisfied, the growth rate $\Gamma=\left(\Upsilon-\Omega^{2}\right)^{1 / 2}$ of the unstable perturbation of these solitary waves is given by

$$
\Gamma=\frac{2}{\sqrt{63}} \frac{u_{0}\left[\left(1+\omega_{c i}^{2} / R^{2}\right) S_{i}\right]^{1 / 2}}{\left(\sin ^{2} \delta+\omega_{c i}^{2} / R^{2}\right) v_{p}^{2}}
$$

Equation (35), with (34) and (35), clearly indicates that the growth rate $\Gamma$ of the unstable perturbation is a linear function of $u_{0}$ but a nonlinear function of $\delta, \omega_{c i}, l_{\zeta}, l_{\eta}, \sigma_{n}$ and $\sigma_{i}$. The nonlinear variations of $\Gamma$ with $\delta, \omega_{c i}, l_{\zeta}, l_{\eta}, \sigma_{n}$ and $\sigma_{i}$. are shown in Figs. 5(a)-(c). Fig. 5(a) shows how the value of $\Gamma$ changes with $\delta$ and $\omega_{c i}$. From this it is clear that the growth rate $\Gamma$ of the unstable perturbation decreases with increasing values of both $\delta$ and $\omega_{c i}$. Fig. $5(\mathrm{c})$ shows how the value of $\Gamma$ changes with $l_{\zeta}$ and $l_{\eta}$. This figure represents that the growth rate $\Gamma$ of the unstable perturbation increases with increasing the values of $l_{\zeta}$ and $l_{\eta}$. Fig. 5(c) shows how the value of $\Gamma$ changes with $\sigma_{n}$ and $\sigma_{i}$. This figure represents that the growth rate $\Gamma$ of the unstable perturbation also increases with increasing the values of $\sigma_{n}$ and $\sigma_{i}$.

\section{Discussion}

In this paper, we have considered a collisionless MADENP containing Boltzmann electrons, vortex-like negative ions, adiabatic mobile positive ions and positively charged stationary dust in the presence of an external static magnetic field, and have studied the DIA SWs associated with positive potential by deriving mZK equation. We have then analyzed their multidimensional instability. The results which have been found in our study are summarized as follows:

1. The SWs associated with positive potential can exist only due to the effects of vortex-like negative ions. It has been also observed that the vortex-like negative ions and positively charged stationary dust particles give rise to stronger non-linearity.

2. The well-known mZK equation is obtained, instead of the ZK equation, due to vortex-like negative ions. The stationary solution of which is represented in the form of $\operatorname{sech}^{4}(k Z)$. Thus, the potential polarity of the DIASWs in this system is different from the usual IA, DA or DIASWs.

3. The trapped particles have no effect on the width of the DIASWs but it increases the amplitude of this waves. The magnitude of the external magnetic field $\mathbf{B}_{0}$ has no any effect on the solitary wave amplitude. However, it does have a direct effect on the width of the solitary waves and the magnitude 
of $\mathbf{B}_{0}$ increases, whereas the width of the SWs decreases, i.e., the magnetic field makes the solitary structures spikier.

4. The soliton speed $u_{0}$ affects the amplitude linearly and the width inversely, the profile of the faster solitary wave will be taller and narrower then slower one. The adiabatic index $\gamma$ increases both the amplitude and width of the DIASWs. It means that the adiabatic effect of positive ions increases the wavelength of the DIASWs.

5. The width of the positive DIASWs potential increases with increasing the values of $\sigma_{i}$, but it decreases with increasing the values of $\mu_{n}, \mu_{d}, \omega_{c i}$ and $\sigma_{n}$. It is also found that the width of the SWs also increases for the lower range of $\delta$ (from $0^{0}$ to about $60^{\circ}$ ) but decreases for its higher range (from $60^{\circ}$ to about $90^{\circ}$ ). The value of $\omega_{c i}$ for which the SWs become unstable increases (decreases) with $l_{\zeta}, \sigma_{n}$, $\sigma_{i}, \mu_{e}, \mu_{n}, \delta$ and $\gamma\left(l_{\eta}\right)$. While the growth rate $\Gamma$ increases with $l_{\eta}, l_{\zeta}, \sigma_{n}$ and $\sigma_{i}$ but decreases with $\delta$ and $\omega_{c i}$.

It has been clear from Eqns. (22)-(24) that if we neglect the effects of the adiabaticity of positive ions, this results will completely agree with the published work [15]. It has also mentioned in this paper that as $\delta \rightarrow 90^{\circ}$, the width goes to zero, and thus amplitude goes to infinity. It is likely that, for large angles, the the assumption that the waves are electrostatic is no longer valid, and we should look for fully electromagnetic structures. It may also, be noted that we have used the reductive-perturbation method and small- $k$ perturbation expansion technique that is valid for small but finite-amplitude SWs and long-wavelength perturbation modes. Since in many astrophysical situations, there are extremely large-amplitude SWs and short-wavelength perturbation modes. A more exact theory should be developed for instability analysis of arbitrary-amplitude SWs and arbitrary-wavelength perturbation modes, through a generalization of this paper to such waves and modes. Therefore, the present results may be useful for understanding the localized electrostatic disturbances in space $[21,22,23]$ and laboratory $[24,25,26]$ plasmas.

\section{References}

1. P. V. Bliokh and V. V. Yaroshenko, "Electrostatic Waves in Saturns Rings", Soviet Astronomy, vol. 29, pp. 330-336, 1985.

2. U. de Angelis, V. Formisano, and M. Giordano, "Ion plasma waves in dusty plasmas: Halley's comet", Journal of Plasma Physics, vol. 40, pp. 399-406, 1988.

3. P. K. Shukla and A. A. Mamun, "Introduction to dusty plasma physics", IoP Publishing Ltd., Bristol, 2002.

4. R. L. Merlino, A. Barkan, C. Thomson, and N. D'Angelo, "Laboratory studies of waves and instabilities in dusty plasmas", Physics of Plasmas, vol. 5, pp. 1607-1614, 1998.

5. P. K. Shukla and V. P. Silin, "Dust ion-acoustic wave", Physica Scripta, vol. 45, pp. 508-508, 1992.

6. A. Barkan, N. D'Angelo, and R. L. Merlino, "Experiment on ion-acoustic waves in dusty plasmas", Planetary and Space Science, vol. 44, pp. 239-242, 1996.

7. A. Barkan, N. D’Angelo, and R. L. Merlino, "Charging of dust grains in a plasma", Physical Review Letters, vol. 73, pp. 3093-3096, 1994.

8. E. C. Whipple, "Potentials of surfaces in space", Reports on Progress in Physics, vol. 44, pp. 1197-1250, 1981.

9. V. W. Chow, D. A. Mendis, and M. Rosenberg, "Role of grain size and particle velocity distribution in Secondary Electron Emission in Space Plasmas", Journal of Geophysical Research, vol. 98, pp. 19065-19076, 1993.

10. D. A. Mendis and M. Rosenberg, "Cosmic dusty plasma", Annual Review of Astronomy and Astrophysics, vol. 32, pp. 419-463, 1994.

11. M. Rosenberg and D. A. Mendis, "UV induced columb crystallization in dusty gas", IEEE Transaction on Plasma Science, vol. 23, pp. 177-179, 1995.

12. M. Horanyi, G. E. Morfill, and E. Grün, "Mechanism for the acceleration and ejection of dust grains from Jupiter's magnetosphere", Nature, vol. 363, pp. 144-146, 1993.

13. V. E. Fortov, A. P. Nefedov, O. S. Vaulina, A. M. Lipaev, V. I. Molotkov, A. A. Samaryan, V. P. Nikitskii, A. I. Ivanov, S. F. Savin, A. V. Kalmykov, A. Ya. Solove'v, and P. V. Vinogradov, "Dusty plasma induced by solar radiation under microgravitational conditions: an experiment on board the Mir orbiting space station", Journal of Experimental and Theoretical Physics, vol. 87, pp. 1087-1097, 1998.

14. F. Sayed, M. M. Haider, A. A. Mamun, P. K. Shukla, B. Eliasson, and N. Adhikary, "Dust ion-acoustic solitary waves in a dusty plasma with positive and negative ions", Physics of Plasmas, vol. 15, pp. 063701-7, 2008. 
15. O. Rahman, A. A. Mamun, and K.S. Ashrafi, "Dust-ion-acoustic solitary waves and their multi-dimensional instability in a magnetized dusty electronegative plasma with trapped negative ion", Astrophysics and Space Science, vol. 335, pp. 425-433, 2011.

16. N.R. Kundu, M.M. Masud, K.S. Ashrafi, and A.A. Mamun, "Dust-ion-acoustic solitary waves and their multi-dimensional instability in a magnetized nonthermal dusty electronegative plasma", Astrophysics and Space Science, vol. 343, pp. 279-,287, 2013.

17. M. M. Haider, T. Ferdous, S. S. Duha, and A. A. Mamun, "Dust-ion-acoustic solitary waves in multi-component magnetized plasmas", Open Journal of Modern Physics, vol. 1, pp. 13-24, 2014.

18. M. M. Haider, T. Ferdous, and S. S. Duha, "Instability due to trapped electrons in magnetized multi-ion dusty plasmas", Journal of Theoretical and Applied Physics, vol. 9, pp. 159-166, 2015.

19. A. A. Mamun, "Effects of adiabaticity of electrons and ions on dust-ion-acoustic solitary waves", Physics Letters A, vol. 372, pp. 1490-1493, 2008.

20. S. S. Duha, M. S. Rahma, A. A. Mamun, and M. G. M. Anowar, "Multidimensional instability of dust ion-acoustic solitary waves in a magnetized dusty electronegative plasma", Journal of Plasma Physics, vol. 78, pp. 279-288, 2012.

21. B. Kozlovsky, R. J. Murphy, and G. H. Share, "Positron-emitter production in solar flares from ${ }^{3}$ He reactions", The Astrophysical Journal, vol. 604, pp. 892-899, 2004.

22. W. H. Lee, E. R. Ruiz and D. Page, "Dynamical evolution of neutrino-cooled accretion disks: Detailed microphysics, Lepton-driven convection, and global energetics", The Astrophysical Journal, vol. 632, pp. 421-437, 2005.

23. T. Piran, "Gamma-ray bursts and the fireball model", Physics Report, vol. 314, pp. 575-667, 1999.

24. C. M. Surko and T. J. Murphy, "Use of the positron as a plasma particle", Physics Fluids B, vol. 2, pp. 1372-1375, 1990.

25. M. Tinkle, R. G. Greaves, C. M. Surko, R. L. Spencer, and G. W. Mason, "Low-order modes as diagnostics of spheroidal non-neutral plasmas", Physical Review Letters, vol. 72, pp. 352-355, 1994.

26. R. G. Greaves and C. M. Surko, "An electron-positron beam-plasma experiment", Physical Review Letters, vol. 75, pp. 3846-3849, 1995.

27. H. Schamel, "Stationary solitary, snoidal and sinusoidal ion acoustic waves", Plasma of Physics, vol. 14, pp. 905-924, 1972.

28. H. Schamel, "A modified Korteweg-de Vries equation for ion acoustic waves due to resonant electrons", Journal of Plasma Physics, vol. 9, pp. 377-387, 1973.

29. H. Schamel, "Analytic BGK modes and their modulational instability", Journal of Plasma Physics, vol. 13, pp. 139-145, 1975.

30. A. A. Mamun, "Solitary waves in a three-component dusty plasma with trapped ions", Physica Scripta, vol. 57, pp. 258-260, 1998.

31. A. A. Mamun, "Nonlinear propagation of dust-acoustic waves in magnetized a dusty plasma with vortex-like ion distribution", Journal of Plasma Physics, vol. 59, pp. 575-580, 1998.

32. M. G. M. Anowar, M. S. Rahman, and A. A. Mamun, "Nonlinear dust-acoustic waves in a strongly coupled dusty plasma with vortexlike ion distribution", Physics of Plasmas, vol. 16, pp. 053704-5, 2009.

33. H. Schamel and S. Bujarbarua, "Solitary plasma hole via ion-vortex distribution", Physics of Fluids, vol. 23, pp. 2498-2499, 1980.

34. A. A. Mamun, B. Eliasson, and P. K. Shukla, "Dust-acoustic solitary and shock waves in a strongly coupled liquid state dusty plasma with a vortex-like ion distribution", Physics Letter A, vol. 332, pp. 412-416, 2004.

35. H. Washimi and T. Taniuti, "Propagation of ion-acoustic solitary waves of small amplitude", Physics Review Letter, vol. 17, pp. 996-997, 1966.

36. M. Shalaby, S. K. EL-Labany, E. F. EL-Shamy, and M. A. Khaled, "Three-dimensional instability of dust ion-acoustic solitary waves in a magnetized dusty plasma with two different types of nonisothermal electrons", Physics of Plasmas, vol. 17, pp. 113709-8, 2010.

37. E. Infeld, "On the stability of nonlinear cold plasma waves", Journal of Plasma Physics, vol. 8, pp. 105-110, 1972.

38. E. Infeld and G. Rowlands, "On the stability of nonlinear cold plasma waves. Part 2", Journal of Plasma Physics, vol. 10, pp. 293-300, 1973.

39. E. Infeld, "Self-focusing of nonlinear ion-acoustic waves and solitons in magnetized plasmas", Journal of Plasma Physics, vol. 33, pp. 171-182, 1985.

40. P. K. Das and F. Verheest, "Ion-acoustic solitons in magnetized multi-component plasmas including negative ions", Journal of Plasma Physics, vol. 41, pp. 139-155, 1989.

41. A. A. Mamun and R. A. Cairns, "Stability of solitary waves in a magnetized non-thermal plasma", Journal of Plasma Physics, vol. 56, pp. 175-185, 1996. 